\title{
An electronic approach to homogenize the impedance phase characteristics of heterogeneous $\mathrm{GMI}$ sensors
}

\author{
Eduardo C. Silva ${ }^{1,2}$, Luiz A.P. Gusmão ${ }^{1}$, Carlos R.H. Barbosa ${ }^{2}$, Elisabeth C. Monteiro ${ }^{2}$ \\ ${ }^{1}$ Department of Electrical Engineering, Pontifícia Universidade Católica do Rio de Janeiro, Rua Marquês de São Vicente 225, 22451-900 Rio \\ de Janeiro, Brazil \\ ${ }^{2}$ Postgraduate Program in Metrology, Pontifícia Universidade Católica do Rio de Janeiro, Rua Marquês de São Vicente 225, 22451-900 Rio \\ de Janeiro, Brazil
}

Over the last decade, Giant Magnetoimpedance (GMI) sensors have been studied as a new possibility for measuring ultra-weak magnetic fields. To achieve that goal, the employment and optimization of gradiometric configurations are a key factor to reduce the magnetic interference. The performance of GMI gradiometers is strongly related to the homogeneity of the impedance characteristics of the GMI samples. This work presents a method and corresponding electronic implementation to homogenize the phase characteristics of heterogeneous GMI samples, aiming at improving the performance of phase-based GMI magnetometers.

Keywords: Homogenization; Impedance Phase; Giant Magnetoimpedance; Magnetic Sensor

Citation: Eduardo C. Silva, Luiz A.P. Gusmão, Carlos R.H. Barbosa, Elisabeth C. Monteiro, An electronic approach to homogenize the impedance phase characteristics of heterogeneous GMI sensors, Acta IMEKO, vol. 1, no. 1, article 14, July 2012, identifier: IMEKO-ACTA-01(2012)-01-14

Editor: Pedro Ramos, Instituto de Telecomunicações and Instituto Superior Técnico/Universidade Técnica de Lisboa, Portugal

Received January $10^{\text {th }}, 2012$; In final form June $5^{\text {th }}, 2012$; Published July 2012

Copyright: () 2012 IMEKO. This is an open-access article distributed under the terms of the Creative Commons Attribution 3.0 License, which permits unrestricted use, distribution, and reproduction in any medium, provided the original author and source are credited

Funding: This work was supported by the Brazilian funding agencies CNPq and FINEP

Corresponding author: Eduardo C. Silva, e-mail: edusilva@ele.puc-rio.br

\section{INTRODUCTION}

The Giant Magnetoimpedance effect (GMI) began to be intensively studied in the 1990s. It was observed a huge impedance variation when a sample of a ferromagnetic amorphous material, excited by an alternating current, was submitted to an external magnetic field [1-19].

The GMI phenomenon is induced by the application of an alternating current $(I)$ along the length of amorphous ferromagnetic samples, which are submitted to an external magnetic field $(H)$ [1-6]. The impedance of GMI samples changes as a function of $H$, as shown in Figure 1, and $H$ can be inferred by measuring the difference of potential $(V)$ between the extremities of the GMI sample.

The complex impedance $Z_{\text {sens }}(H)$ of the GMI samples can be electrically modelled as a resistor $R_{\text {sens }}(H)$ in series with an inductor $L_{\text {sens }}(H)$, as defined by (1).

$Z_{\text {sens }}(H)=R_{\text {sens }}(H)+j w L_{\text {sens }}(H)$,

where

$$
\begin{aligned}
& \mathrm{R}_{\text {sens }}(H)=\left|Z_{\text {sens }}(H)\right| \cos \theta_{\text {sens }}(H) \text { and } \\
& w L_{\text {sens }}(H)=\left|Z_{\text {sens }}(H)\right| \sin \theta_{\text {sens }}(H) .
\end{aligned}
$$

where $\theta_{\text {sens }}$ is the impedance phase of the GMI samples.

Over the last years, the research group of the Laboratories of Sensors and Instrumentation (LSI) and Biometrology (LaBioMet) of PUC-Rio have worked on the development of transducers based on the GMI effect [10-19]. Recent researches, conducted by the LSI and LaBioMet team allowed observing that phase-based GMI transducers are much more sensitive than the usual magnitude-based GMI transducers [14-19].

Besides, a newly developed electronic circuit capable of improving by about a hundred times the impedance phase sensitivity of the phase-based GMI transducers [17] allowed to envision the application of the GMI magnetometers for the

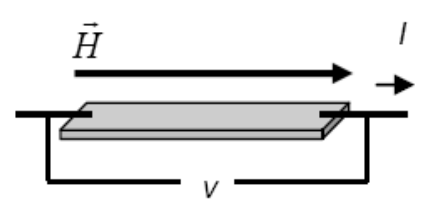

Figure 1. Typical measurement of the GMI effect: The difference of potential $(V)$ between the extremities of a GMI sample, submitted to an excitation current $(I)$, as a function of the magnetic field $(H)$. 
measurement of ultra-weak magnetic fields, such as biomagnetic fields [5, 7-9, 20-21].

By definition, biomagnetic fields are magnetic fields generated by living organisms or by magnetic markers placed on these organisms. The intensities of the magnetic flux densities of biomagnetic fields are comprised between $1 \mathrm{fT}$ and $1 \mathrm{nT}$, and their frequencies are in the range of $\mathrm{DC}$ to $1 \mathrm{kHz}$ [20-21]. Among all the magnetometers (fluxgates, Hall Effect, magnetoresistance, etc.), the Superconducting Quantum Interference Devices (SQUID) are the most sensitive, and the only ones capable of satisfactorily performing biomagnetic measurements. However, SQUID systems need cryogenic cooling, presenting the drawback of high cost of fabrication, installation and operation [5-6, 20-21]. This drawback of the SQUID systems hinders the broad dissemination of an important non-invasive diagnostic tool based on biomagnetic field measurements [5, 20-21].

High sensitivity magnetic transducers, capable of measuring ultra-weak magnetic fields, have their resolutions limited by spurious magnetic interference due to diverse sources (vehicles, elevators, Earth's magnetic field, etc.) which, in a typical laboratory environment, is in the order of $10 \mathrm{nT}$ in the neighbourhood of $1 \mathrm{~Hz}$ [5, 20-21]. Thus, in principle, no matter how sensitive a transducer is, it cannot detect magnetic fields below the noise/interference level.

To solve that issue, there are two usual approaches: magnetic shielding and gradiometric configurations. In spite of its high attenuation of the magnetic noise and interference, magnetic shields are very expensive, especially when it is necessary to filter low frequency fields, which significantly affect the measurements of biomagnetic fields, because it requires the use of materials with high magnetic permeability at low frequencies, like $\mu$-metal alloys [20-21].

On the other hand, the gradiometric configuration approach, which consists in the use of spatial filters, is a much cheaper alternative, and also effective in most applications. Since the magnetic field of a current dipole decreases with the cube of the distance $r$ between this source and the point of measurement, the gradient field detection using two sensor elements separated by a distance $d$, significantly weakens the contribution of magnetic interference sources located at $r>>d$ in relation to the contribution of the closest signal source at $r<<d$ [20-21].

The performance of gradiometric configurations is highly dependent of the sensors homogeneity [20-21]. In this work, aiming at the future implementation of GMI gradiometers, the heterogeneity of impedance phase characteristics of GMI samples is experimentally analysed and a method to homogenize the GMI sensors behaviour is developed. It is also proposed and discussed the electronic implementation of the homogenization method.

\section{IMPEDANCE CHARACTERIZATION}

The GMI sensors analyzed in this work are ribbon-shaped samples of $\mathrm{CO}_{70} \mathrm{Fe}_{5} \mathrm{Si}_{15} \mathrm{~B}_{10}$, produced by the Single-Roller MeltSpinning technique. These samples show a particular case of the GMI effect, called Longitudinal Magnetoimpedance (LMI). LMI samples are much more sensitive to the component of the magnetic field parallel to its length.

The sensitivity of the GMI samples is typically affected by some parameters such as amplitude, frequency and DC level of excitation current, dimensions (length, width, thickness) of the GMI samples; biasing magnetic field (generated by an external source to ensure that the sensor operates in its most sensitive range), among others. Therefore, the influence of these parameters under the GMI effect was experimentally investigated in order to ascertain which is the set of parameters that generates the optimum sensitivities [1-4].

Thus, the behaviour of the impedance magnitude $\left|Z_{\text {sens }}(H)\right|$ and phase $\theta_{\text {sens }}(H)$ of the ribbon-shaped GMI sensors, as a function of the external magnetic field $H$, was analyzed under different conditions. It was studied the dependency in relation to the excitation currents of the samples for DC levels between $0 \mathrm{~mA}$ and $100 \mathrm{~mA}$, and frequencies between $75 \mathrm{kHz}$ and 30 $\mathrm{MHz}$. Also, it was verified that the amplitude of the AC component of the excitation current does not significantly affect the impedance behaviour, so it was kept in $15 \mathrm{~mA}$. The influence of the length of the GMI sensors was also investigated. The measurements were performed with four different lengths: $1 \mathrm{~cm}, 3 \mathrm{~cm}, 5 \mathrm{~cm}$ and $15 \mathrm{~cm}$ [14-19].

The analysed GMI samples were put in the centre of a Helmholtz coil, as shown in Figure 2, in order to be excited by a magnetic field longitudinal to the direction of the current that flows through them. Also, the sample-coil set was positioned in a way to guarantee that the direction of the Earth's magnetic field was transversal to the ribbons-shaped GMI samples. Thus, its influence in the measurements has been minimized, because the GMI sensors used are of the LMI type.

The variation of the magnetic field generated by the Helmholtz pair was obtained by a DC current source according to

$H(\mathrm{Oe})=2.877 \times I(\mathrm{~A})$.

The impedance measurements of the GMI samples, analyzed during the characterization studies, were performed by the preciion LCR meter Agilent 4285A, which was also responsible for both AC and DC excitation of the samples. The uncertainty of the impedance phase measurements of the samples $U_{\theta}$ is directly related to the impedance phase uncertainty of the LCR meter, which is indicated in its operational manual, in degrees, as

$U_{\theta}= \pm \frac{180 \times A_{e}}{\pi \times 100}$

where $A_{e}$ is defined as

$A_{e}(\%)= \pm\left(A_{n}+A_{c}\right) \times K_{t}$,

where $A_{n}$ is the component of the uncertainty due to the equipment intrinsic characteristics, $A_{c}$ is the cable length factor and $K_{t}$ is the temperature factor.

The temperature factor $K_{t}$ is equal to one in the range of $18^{\circ} \mathrm{C}$ to $28^{\circ} \mathrm{C}$. The measurements were always performed

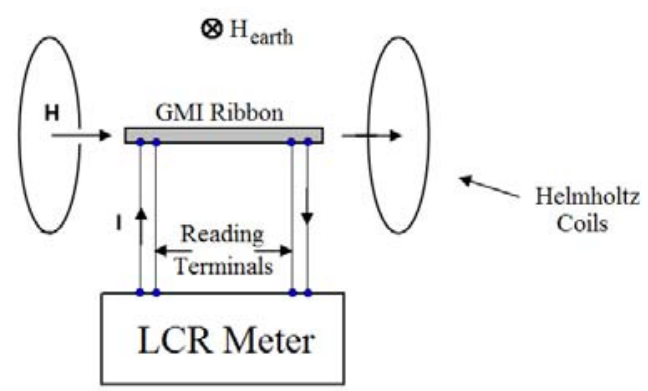

Figure 2. Block diagram of the system used in the experimental characterization of the ribbon-shaped GMI samples. 
within this temperature range, then it can be admitted that $K_{t}=$ 1. On the other hand, for impedance magnitudes below $5 \mathrm{k} \Omega$, $A_{c}$ is given by

$$
A_{c}(\%)=\frac{f_{m}}{15}
$$

where $f_{m}$ is the frequency, in $\mathrm{MHz}$, used to excite the sample.

Then, knowing that all of the experimental measurements of the impedance magnitudes of the GMI sensors returned values between $10 \mathrm{~m} \Omega$ and $100 \Omega$, the parameter $A_{n}$ is defined as

$$
A_{n}(\%)=N_{2} \%+\left(\frac{f_{m}}{30}\right)^{2} \times 3 \%+\frac{100}{\left|Z_{m}\right|}\left[0.02 \%+\left(\frac{f_{m}}{30}\right) \times 0.1 \%\right],(8)
$$

where $\left|Z_{m}\right|$ is the absolute value of the measured impedance in ohms and $N_{2}$ is a frequency-dependent factor which can be equal to 0.15 - frequencies between $75 \mathrm{kHz}$ and $3 \mathrm{MHz}$ - or to 0.38 - frequencies above $3 \mathrm{MHz}$.

The standard uncertainty of the magnetic field $\left(u_{H}\right)$ generated by the Helmholtz pair is dependent of the standard uncertainty of the DC current source (ICEL, PS-4000), which is equal to $\pm 3.25 \mathrm{~mA}$. Then, supposing, by simplicity, that the geometric configuration of the Helmholtz Coils is satisfactorily close to the one considered on the theoretical model and knowing that the relation between the current and the magnetic field generated by the Helmholtz Pair is given by (4), $u_{H}$ is expressed as

$u_{H}(\mathrm{Oe})= \pm 2.877 \times u_{I}(\mathrm{~A})= \pm 9.35 \mathrm{mOe}$.

Thus, the expanded uncertainty $U_{H}$, for a confidence level of $95.45 \%$, is

$U_{H}=2 \times u_{H}= \pm 18.70 \mathrm{mOe}$.

The oersted (Oe) is the unit of magnetic field in the CGS system, which is broadly used in the giant magnetoimpedance literature. The SI unit (International System of Units) of magnetic field is ampere per meter $\left(\mathrm{Am}^{-1}\right)$, which is related to the oersted by

$$
1 \mathrm{Oe}=\frac{1}{4 \pi} \times 10^{3} \mathrm{Am}^{-1} .
$$

\section{PHASE HOMOGENIZATION METHOD}

Assuming two heterogeneous GMI sensor elements ( $A$ and $B$ ), with different behaviours of the impedance phase as a function of the magnetic field $(H)$, this section describes a method that allows the homogenization of the impedance phase behaviour of such sensors in a given range of magnetic fields $\left(H_{1}\right.$ to $\left.H_{2}\right)$.

The sensor $B$ should be inserted in an "homogenizer circuit", shown in Figure 3, in order to generate an equivalent impedance $Z_{B}$ such that the phase of $Z_{B}$ in $H_{1}$ and $H_{2}\left(\theta_{1 B}\right.$ and $\theta_{2 B}$ ) becomes equal to the phase of the sensor $A$ in these same points $\left(\theta_{1 A}\right.$ and $\left.\theta_{2 A}\right)$. Regions should be chosen preferably where there is a linear, or almost linear, relationship between the phase of the GMI sensors and the magnetic field, so that the homogenization procedure can be optimal.

When the sensor $B$ is connected to the "homogenizer circuit" (Figure 3), it is created an equivalent impedance $Z_{B}$, which phase characteristics behaves similarly to the ones of sensor $A$, within a given magnetic field range. The block reactance shown in Figure 3 may be replaced by an inductance

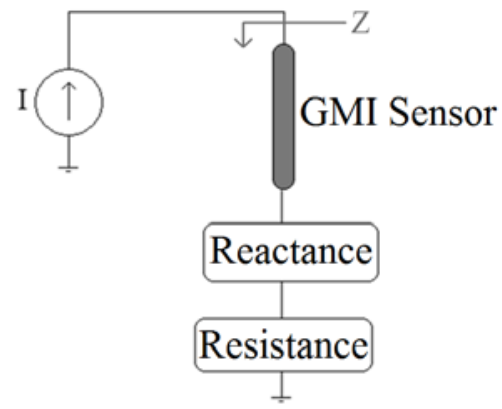

Figure 3. Block diagram of the "homogenizer circuit".

$\left(L_{a d j}\right)$ or capacitance $\left(C_{a d j}\right)$ as required, and the block resistance may represent a positive or negative resistance depending on the case. It is emphasized that despite the difficulties to implement a negative resistance using passive elements, it is possible to reproduce its behaviour by means of active electronic structures called GIC's (Generalized Immittance Converters) [22].

Among the possible implementations of GIC's the one that uses two coupled OpAmp's, as shown in Figure 4a, is considered the best, due to the fact that it is very stable and highly tolerant to nonideal properties of the OpAmp's, particularly its finite gain and limited bandwidth [22].

One can infer that a GIC can be used to simulate a negative resistance by adequately choosing the impedances $Z_{i}$ that compose the GIC structure. These circuits are called FDNR's (Frequency-Dependent Negative-Resistance), because the generated negative resistances are dependent of the frequency. Among the implementations of FDNR's, the most recommended is shown in Figure 4b [22].

The input equivalent impedance $\left(Z_{G I C}\right)$ of the GIC shown in Figure $4 \mathrm{a}$, assuming ideal operational amplifiers, is given by

$Z_{\text {GIC }}=\frac{Z_{2} Z_{4} Z_{6}}{Z_{3} Z_{5}}$

and the equivalent impedance $\left(Z_{F D N R}\right)$ of the FDNR shown in Figure $4 \mathrm{~b}$ is given by

$Z_{F D N R}=-\frac{R_{4}}{w^{2} C_{2} C_{6} R_{3} R_{5}} \stackrel{R_{3}=R_{4}}{\longrightarrow} Z_{F D N R}=-\frac{1}{w^{2} C_{2} C_{6} R_{5}}$.

By considering a reactance $X_{a d j}$ (inductive or capacitive) and a resistance $R_{a d j}$ (positive or negative), which will be placed in series with the sensor $B$, in order to homogenize the phase

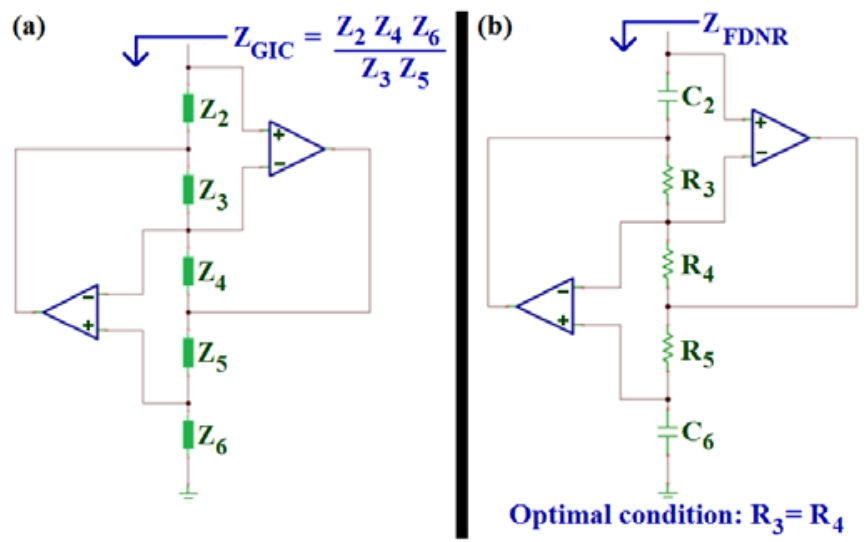

Figure 4. (a) Generalized Immittance Converters (GIC) and (b) FrequencyDependent Negative-Resistance (FDNR). 
characteristics one should attend

$$
\left\{\begin{array}{l}
\theta_{1 A}=\theta_{1 B} \Rightarrow \frac{X_{1 A}}{\mathrm{R}_{1 A}}=\frac{X_{1 B}+X_{a d j}}{\mathrm{R}_{1 B}+\mathrm{R}_{a d j}} \Rightarrow \\
\Rightarrow X_{1 A}\left(\mathrm{R}_{1 B}+\mathrm{R}_{a d j}\right)=\mathrm{R}_{1 A}\left(X_{1 B}+X_{a d j}\right) \\
\theta_{2 A}=\theta_{2 B} \Rightarrow \frac{X_{2 A}}{\mathrm{R}_{2 A}}=\frac{X_{2 B}+X_{a d j}}{\mathrm{R}_{2 B}+\mathrm{R}_{a d j}} \Rightarrow \\
\Rightarrow X_{2 A}\left(\mathrm{R}_{2 B}+\mathrm{R}_{a d j}\right)=\mathrm{R}_{2 A}\left(X_{2 B}+X_{a d j}\right) .
\end{array}\right.
$$

By solving the system of equations presented in (14), it is highlighted that $X_{a d j}$ may yield either a positive value (meaning that $X_{a d j}$ is an inductance $L_{a d j}$ ) or a negative value (meaning that $X_{a d j}$ is a capacitance $\left.C_{a d j}\right)$, leading to

$$
L_{a d j}=\frac{X_{a d j}}{w} \text { or } C_{a d j}=-\frac{1}{w X_{a d j}} .
$$

\section{RESULTS AND DISCUSSION}

Figure 5 shows the phase characteristics of two GMI ribbon-shaped sensor elements $(A$ and $B)$, both of them $3 \mathrm{~cm}$ long and submitted to the same excitation current - DC level of $80 \mathrm{~mA}, 15 \mathrm{~mA}$ of amplitude and $100 \mathrm{kHz}$ of frequency. All of the impedance phase values shown in this section have their respective measurement uncertainties $U_{\theta}$, obtained by applying (5), equal or smaller than \pm 1 .

In turn, Figures 6 and 7 represent, respectively, the dependence of the resistance $\left(R_{\text {senn }}\right)$ and inductance $\left(L_{\text {sens }}\right)$, as a function of magnetic field $(H)$, for the same GMI sensors $A$

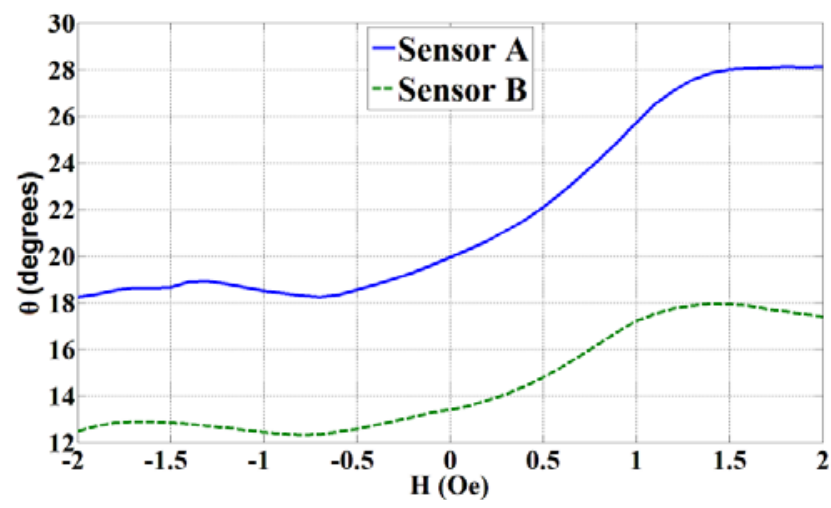

Figure 5. Impedance phase characteristics, of heterogeneous GMI samples $(A$ and $B)$, as a function of the external magnetic field.

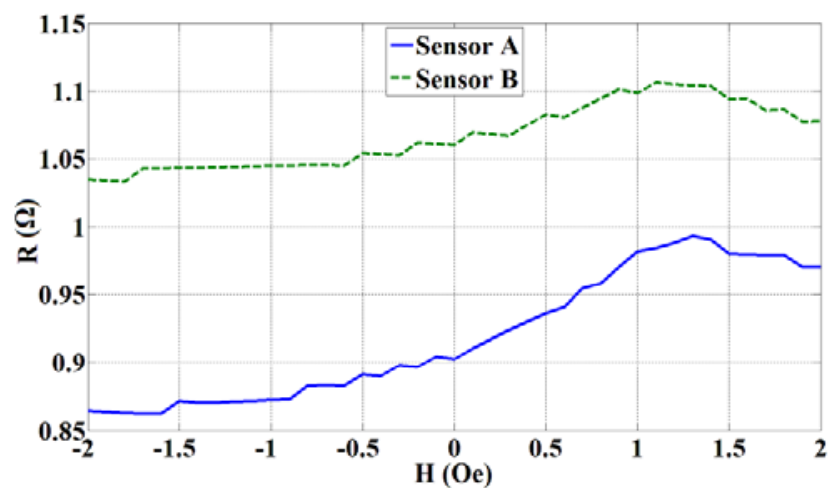

Figure 6. Characteristics of the resistive component of the impedance, of heterogeneous $\mathrm{GMI}$ samples $(A$ and $B$ ), as a function of the external magnetic field.

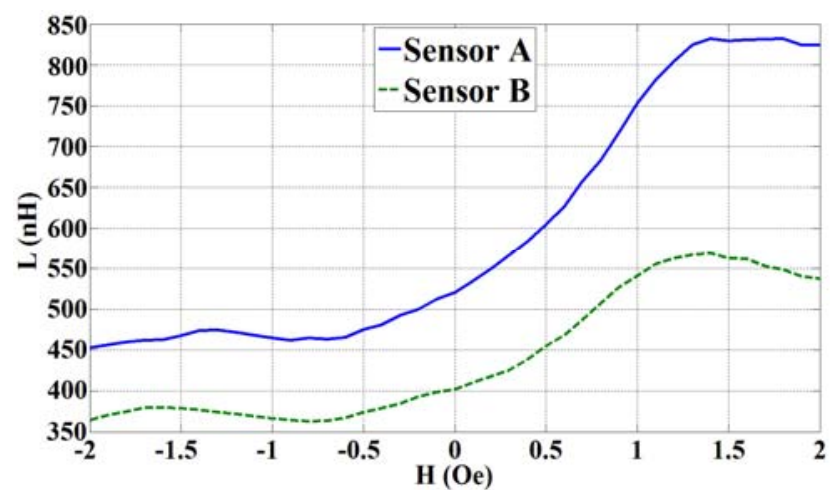

Figure 7. Characteristics of the reactive (inductive) component of the impedance, of heterogeneous GMI samples $(A$ and $B)$, as a function of the external magnetic field.

and $B$ whose phase characteristics are presented in Figure 5.

Sensors $A$ and $B$ are clearly heterogeneous, then it is aimed to make the phase behaviour of sensor $B$ similar to the phase behaviour of sensor $A$, in the region between $H_{1}=0.3$ Oe and $H_{2}=1.0$ Oe. Thus, the homogenization can be accomplished by applying the experimental data of $R_{\text {sens }}$ and $L_{\text {sens }}$, obtained from sensors $A$ and $B$, on the system of equations defined in (14). The following parameters of interest were used: $R_{1 A}=$ $0.9237 \Omega, L_{1 A}=566.7 \mathrm{nH}, R_{2 A}=0.9819 \Omega, L_{2 A}=753.1 \mathrm{nH}$, $R_{1 B}=1.0670 \Omega, L_{1 B}=425.6 \mathrm{nH}, R_{2 B}=1.0990 \Omega, L_{2 B}=$ $541.2 \mathrm{nH}$. With these parameters it is possible to solve the system given by (14), obtaining

$$
\left\{\begin{array}{l}
\mathrm{R}_{a d j}=-0.4737 \Omega \\
X_{a d j}=-0.0387 \Omega \Rightarrow C_{a d j}=-\frac{1}{w X_{a d j}}=41.12 \mu \mathrm{F}
\end{array}\right.
$$

Figure 8 compares the phase characteristics of sensor $A$ with the ones of the equivalent impedance $Z_{B}$, generated by the insertion of sensor $B$ in the electronic circuit of Figure 3, using the reactance and resistance values given by (16).

On the other hand, Figure 9 shows the point-to-point error, in degrees, between the phase curves, shown in Figure 8, as a function of the magnetic field. The analysis in Figure 9 is presented for the region of magnetic fields where the homogenization was applied (0.3 Oe to 1.0 Oe). By observing Figures 8 and 9 it is possible to note that the proposed procedure of homogenization was fairly successful, generating satisfactorily alike phase characteristics.

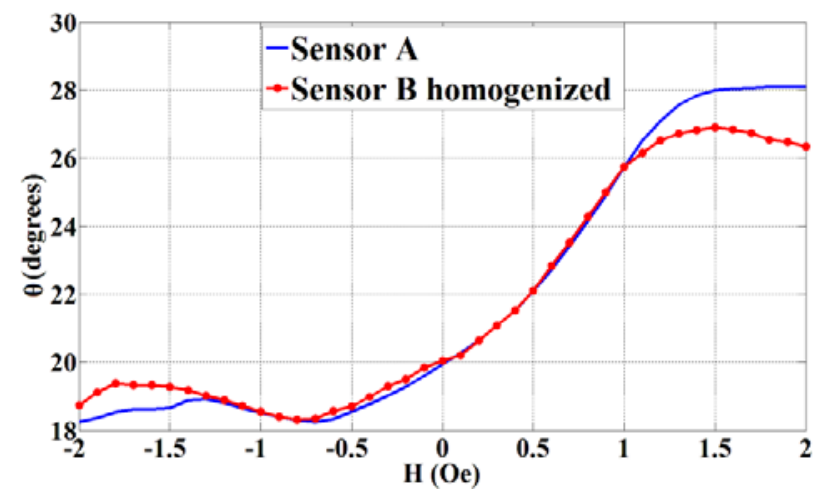

Figure 8. Comparison between the impedance phase characteristics of sensor $A$ and sensor $B$, with sensor $B$ connected to the "homogenizer circuit". 


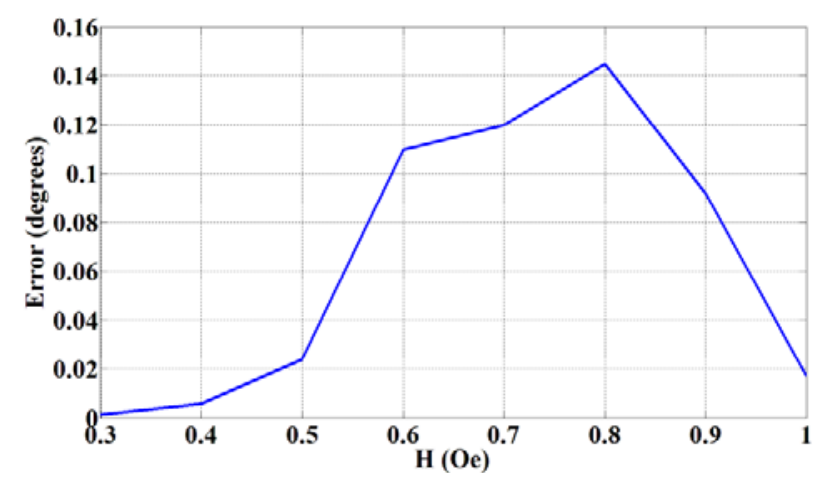

Figure 9. Error in the homogenized region ( 0.3 Oe to $1.0 \mathrm{Oe}$ ) between the phase curves of sensor $A$ and sensor $B$, when the last one is connected to the "homogenizer circuit".

Comparisons between the resistive components of sensors $A, B$, and $B$ connected to the "homogenizer circuit" and between their inductive components are shown, respectively, in Figures 10 and 11. At first notice, it could be expected that inserting the sensor $B$ in the "homogenizer circuit" would lead to having their resistive and inductive components similar to those presented by sensor $A$. However, by observing Figures 10 and 11 , it is noticed that this did not happen.

Actually, that did not happen because the aim of the homogenization process is having as close as possible (homogenize) the phase characteristics of heterogeneous sensors, in a defined magnetic field region, which does not necessarily implies in also homogenizing the characteristics of the resistive and reactive components of the sensors.

\subsection{Sensitivity Analysis}

Aiming at knowing the influence of the adjustment parameters $\left(R_{a d j}\right.$ and $\left.C_{a d j}\right)$ in the homogenization results, a sensitivity analysis was performed. This analysis consists in changing only one parameter (input) at a time, while all other parameters are assumed as constants, and observe how it affects the results (output).

First, it was analysed the effect of variations of $\pm 10 \%$ around the theoretical value obtained for $C_{\text {adj }}$ - equation (16). The results are presented in Figure 12. It is highlighted that $10 \%$ was selected because this is a usual tolerance value for ceramic capacitors. It is also important to note that, in practice, it is difficult to precisely set capacitance values of the order of

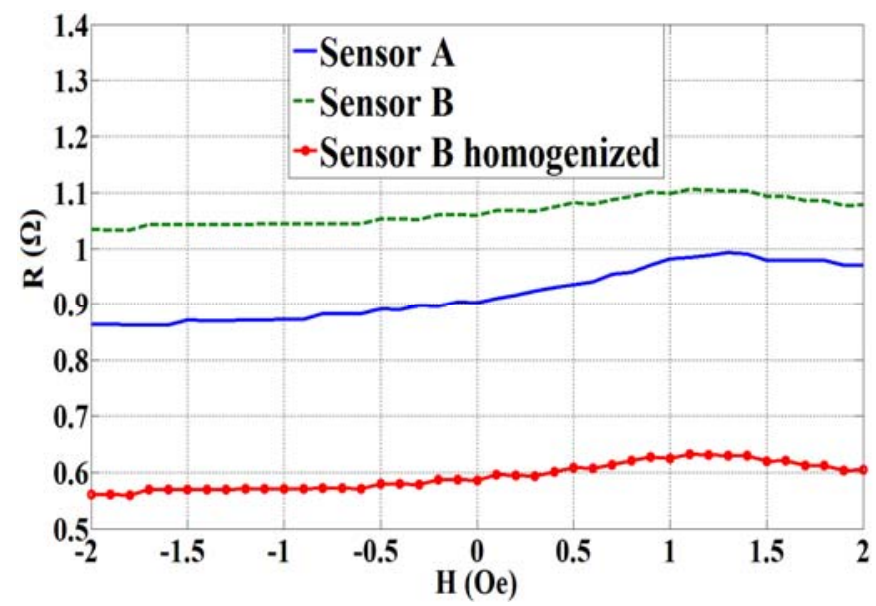

Figure 10. Comparison between the resistive components of the GMI sensors: $A, B$ and $B$ connected to the "homogenizer circuit".

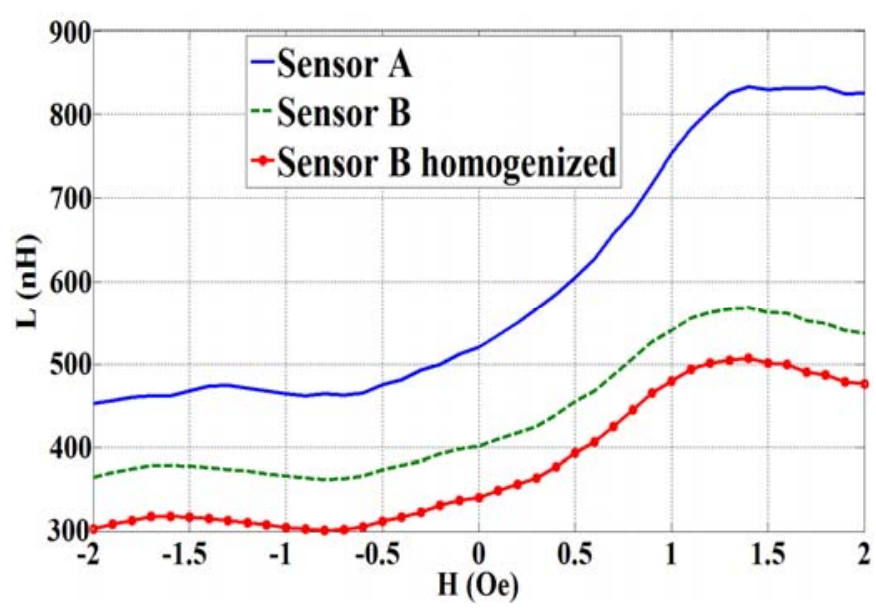

Figure 11. Comparison between the inductive components of the $\mathrm{GMI}$ sensors: $A, B$ and $B$ connected to the "homogenizer circuit".

tenths of $\mu \mathrm{F}$, then it is desirable that these changes in $C_{a d j}$ do not affect significantly the homogenization results.

Second, it was analyzed the effect of variations of $\pm 10 \%$ around the theoretical value obtained for $R_{a d j}$ - equation (16). These results are shown in Figure 13. Once the value of $R_{\text {adj }}$ is set by properly adjusting the internal impedances of an FDNR structure - equation (13) - it is relatively easy to guarantee the correct adjustment of that parameter.

Despite the frequency being not an adjustment parameter, it can affect the homogenization once the negative resistance implemented by FDNR's depends on the frequency - equation (13) - and the ideal value for $C_{\text {alj }}$ also depends on the frequency - equation (15). Thus, a sensitivity analysis was performed, varying the frequency $\pm 10 \%$ around its theoretical value $(100 \mathrm{kHz})$, in order to observe the influence of that parameter in the homogenization, and the results are presented in
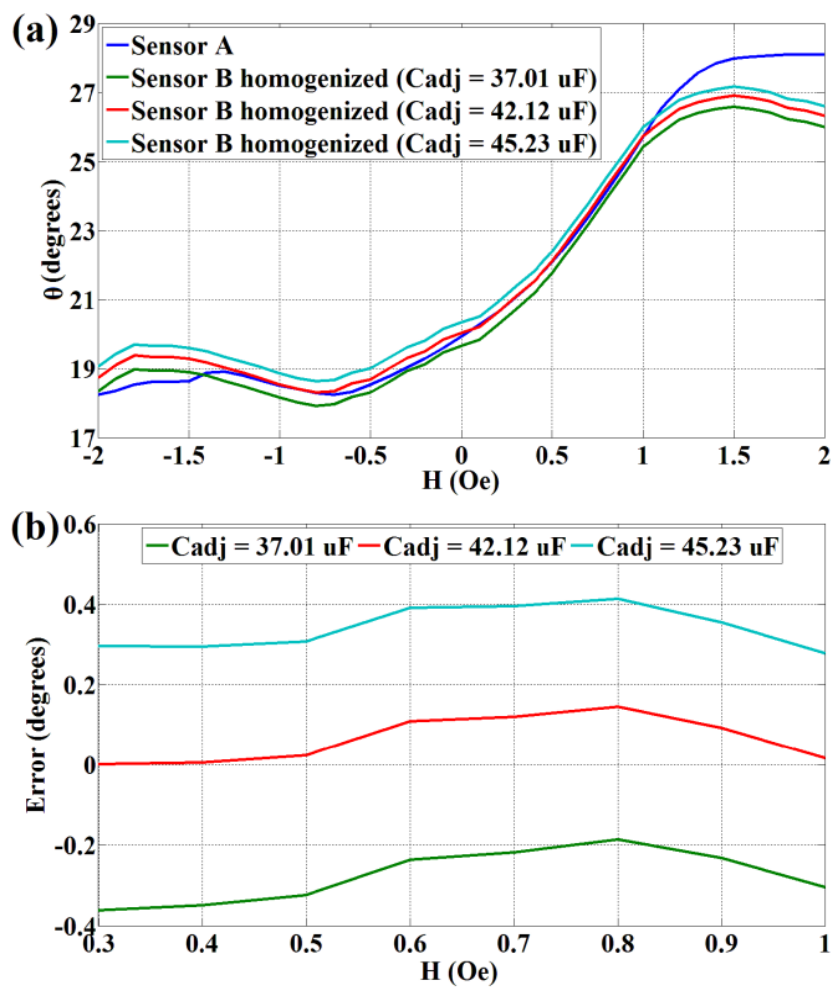

Figure 12. Influence of $C_{a d j}$ in the homogenization curves: (a) Impedance phase characteristics and (b) Error in the homogenized region. 


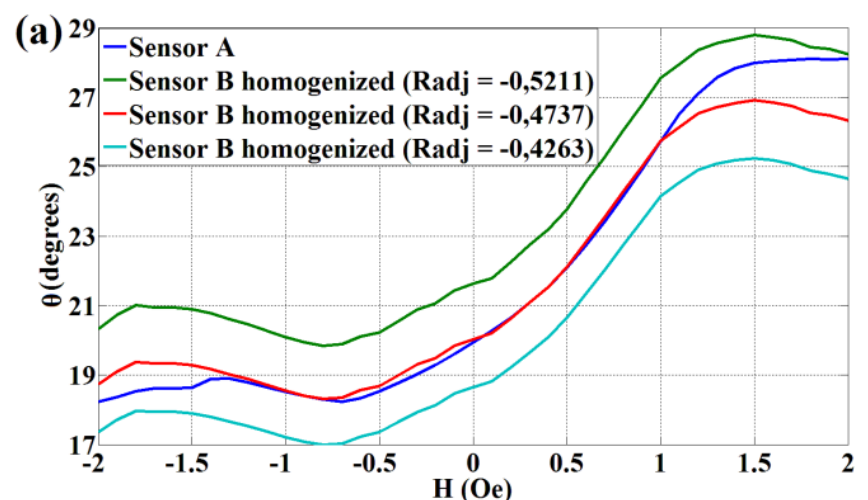

(b) ${ }^{2}$

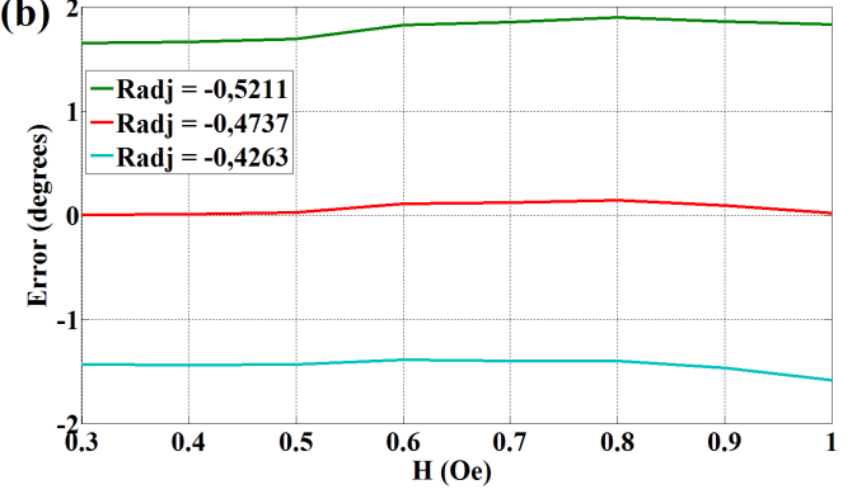

Figure 13. Influence of $R_{a d j}$ in the homogenization curves: (a) Impedance phase characteristics and (b) Error in the homogenized region.

Figure 14. It should be mentioned that sinusoidal oscillators of $100 \mathrm{kHz}$ with uncertainties much smaller than $\pm 10 \%$ are very common.

Figure 12 allows observing that $C_{\text {adj }}$ does not significantly
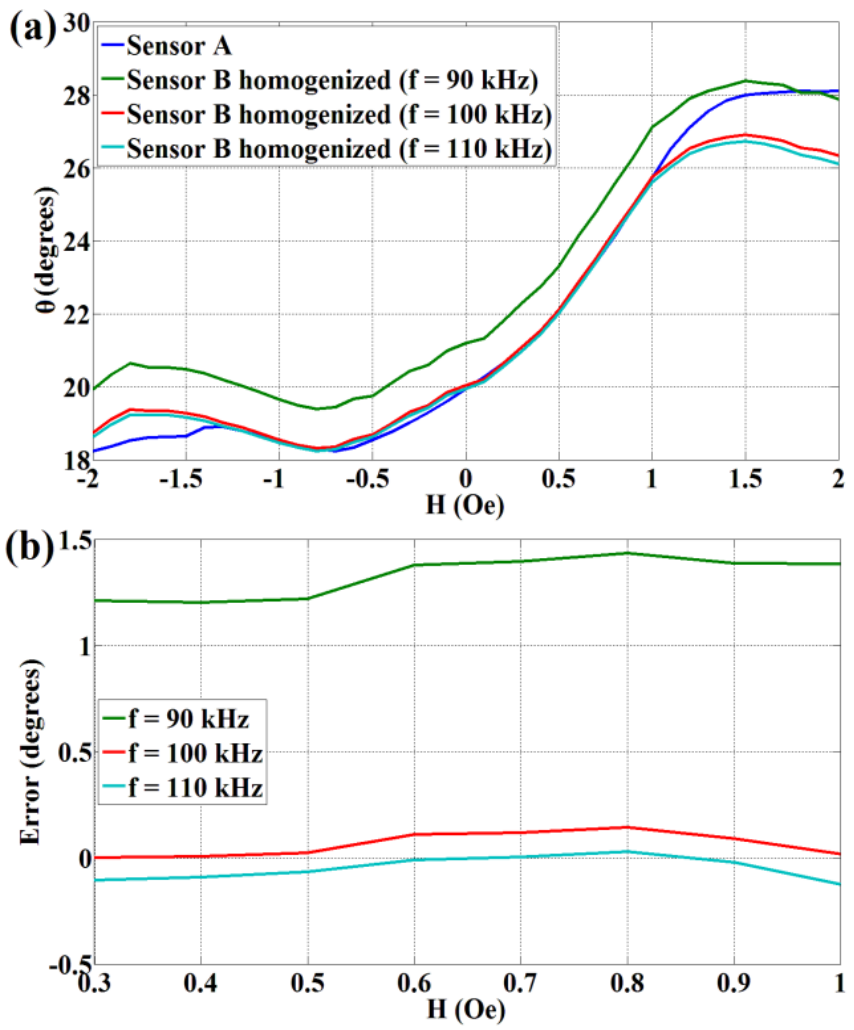

Figure 14. Influence of the frequency $f$ in the homogenization curves: (a) Impedance phase characteristics and (b) Error in the homogenized region. affects the homogenization, even for variations of the order of $10 \%$ around its theoretical value $(41.12 \mu \mathrm{F})$, which is extremely desirable. On the other hand, $R_{a d j}$ has a much larger influence on the results, as shown in Figure 13, pointing that $R_{a d j}$ should be precisely settled. This fact is somewhat preferable because, in practice, as mentioned previously, it is easier to adjust $R_{a d j}$ than $C_{\text {adj }}$.

About the frequency, the results shown in Figure 14 lead to conclude that it affects the results in a nonlinear way. Then, the sinusoidal oscillator must be carefully chosen to minimize these undesirable effects.

\subsection{Alternative Homogenization Approach}

It is highlighted that, in the region of interest ( 0.3 Oe to $1.0 \mathrm{Oe})$, sensor $A$ has a higher sensitivity than sensor $B$. The applied method aimed at making the phase behaviour of sensor $B$ similar to the one presented by sensor $A$, so it was concluded that one should use a capacitor and a negative resistance in series with sensor $B$. However, if the purpose was the opposite, i.e. making the phase behaviour of sensor $A$ similar to the one presented by $B$, it should be used a positive resistance and an inductor in series with sensor $A$. More specifically, in that case, solving (14) leads to

$\left\{\begin{array}{l}\mathrm{R}_{\text {adj }}=0.762 \Omega, \\ X_{a d j}=0.06641 \Omega \Rightarrow L_{a d j}=\frac{X_{a d j}}{w}=105.7 \mathrm{nH} .\end{array}\right.$

This alternative approach presents homogenization results as good as the ones obtained with the original method. In practical terms, this approach is simpler than the original one, because it does not need a negative resistance. However, it reduces the sensitivity of the homogenized sample, which is a drawback. Thus, it should be used only when sensitivity is not a critical factor.

\section{CONCLUSIONS}

This paper presents a new method capable of homogenizing the impedance phase characteristics of heterogeneous GMI sensors. It is also described an electronic circuit, called "homogenizer circuit", which can be used on the implementation of the proposed method.

The results obtained demonstrate the effectiveness of the developed homogenization method, as shown in Figures 8 and 9, allowing that GMI sensors with different phase behaviours, after being inserted in the developed "homogenizer circuit", evolve to almost homogeneous phase behaviours. Within the homogenized region, the difference between the phase characteristics of the sensors was always lower than $0.15^{\circ}$.

In future work, a gradiometric configuration will be implemented using the developed "homogenizer circuit", aiming at improving the quality of the gradiometric readings and, consequently, enhancing the performance of phase-based GMI magnetic transducers.

\section{ACKNOWLEDGEMENT}

We thank the Brazilian funding agencies CNPq and FINEP, for their financial support, and Prof. Fernando Luis de Araújo Machado (Department of Physics / UFPE) for the GMI samples provided. 


\section{REFERENCES}

[1] L.A.P.Gonçalves, J.M.Soares, F.L.A.Machado, W.M.de Azevedo, GMI effect in the low magnetostrictive CoFeSiB alloys, Physica B 384 (2006) pp.152-154.

[2] V.Knobel, K.R.Pirota, Giant magnetoimpedance concepts and recent progress, J. Magn. Magn. Mater. 242 (2002) pp.33-40.

[3] H.Hauser, L.Kraus, P.Ripka, Giant magnetoimpedance sensors, IEEE Instrum. Meas. Mag. 4(2) (2001) pp.28-32.

[4] M.H.Phan, H.X.Peng, Giant magnetoimpedance materials: Fundamentals and applications, Prog. Mat. Sci. 53 (2008) pp.323-420.

[5] A.E.Mahdi, L.Panina, D.Mapps, Some new horizons in magnetic sensing: high-Tc SQUIDs, GMR and GMI materials, Sensors and Actuators A 105 (2003) pp.271-285.

[6] J.Lenz, A.S.Edelstein, Magnetic sensors and their applications, IEEE Sensors Journal, 6(3) (2006) pp.631-649.

[7] T. Uchiyama, S.Nakayama, K.Mohri, K.Bushida, Biomagnetic field detection using very high sensitivity magnetoimpedance sensors for medical applications, Phys. Status Solidi A 206(4) (2009) pp. 639-643.

[8] H.Chiriac, M.Tibu, A.E.Moga, D.D.Herea, Magnetic GMI sensor for detection of biomolecules, J. Magn. Magn. Mater. 293 (2005) pp. 671-673.

[9] K.Totsu, Y.Haga, M.Esashi, Three-axis magnetoimpedance effect sensor system for detecting position and orientation of catheter tip, Sens. Actuators A 111 (2004) 304-309.

[10] C.R.H.Barbosa, E.C.Monteiro, F.M.P.Cavalcanti, "Localization of Magnetic Foreign Bodies in Humans using Magnetic Field Sensors", Proc. of XVII IMEKO World Congress - Metrology in the 3rd Millennium, Dubrovnik, Croatia, 2003, p.1510-1513.

[11] F.Pompéia, L.A.P.Gusmão, C.R.H.Barbosa, E.C.Monteiro, L.A.P.Gonçalves, F.L.A.Machado, "Development of a Low Intensity Magnetic Field Transducer for Biomedical Application based on the Giant Magnetoimpedance Effect", Proc. of Joint International IMEKO TC1+ TC7 Symposium, Ilmenau, Germany, 2005.

[12] D.R.Louzada, E.C.Monteiro, L.A.P.Gusmão, C.R.H.Barbosa, "Medição não-invasiva de ondas de pulso arterial utilizando transdutor de pressão MIG", Proc. of IV Latin American Congress on Biomedical Engineering, Isla de Margarita, Venezuela, 2007, pp. 436-439.

[13] F.M.P.Cavalcanti, L.A.P.Gusmão, C.R.H.Barbosa, E.C.Monteiro, L.A.P.Gonçalves, F.L.A.Machado, Ring shaped magnetic field transducer based on the GMI effect, Measurement Science \& Technology, 19 (2008) pp.1-10.

[14] E.C.Silva, L.A.P.Gusmão, C.R.H.Barbosa, E.C.Monteiro, "Magnetic field transducers based on the phase characteristics of GMI sensors and aimed to Biomedical Applications", Proc. of 13th ICBME, Singapore, 2009, pp. 652-656.

[15] E.C.Silva, L.A.P.Gusmão, C.R.H.Barbosa, E.C.Monteiro, Transdutor de pressão, baseado nas características de fase do efeito GMI, destinado a aplicações biomédicas, Controle \& Automação, 21(6) (2010) pp. 598-608.

[16] E.C.Silva, L.A.P.Gusmão, C.R.H.Barbosa, E.C.Monteiro, F.L.A.Machado, High sensitivity giant magnetoimpedance (GMI) magnetic transducer: magnitude versus phase sensing, Measurement Science \& Technology, 22 (2011) pp.1-9.

[17] E.C.Silva, L.A.P.Gusmão, C.R.H.Barbosa, E.C.Monteiro, "Progress Toward a Hundredfold Enhancement in the Impedance Phase Sensitivity of GMI Magnetic Sensors aiming at Biomagnetic Measurements", Proc. of the V Latin American Congress of Biomedical Engineering, Havana, Cuba, 2011.

[18] PUC-Rio. Pontifícia Universidade Católica do Rio de Janeiro. E.C.Silva, L.A.P. Gusmão, C.R.H.Barbosa, E.C.Monteiro, F.L.A.Machado, F.Pompéia, D.R.Louzada, Patent, BR PI 0902770-0, February 17, 2009.

[19] PUC-Rio. Pontifícia Universidade Católica do Rio de Janeiro. E.C.Silva, L.A.P.Gusmão, C.R.H.Barbosa, E.C.Monteiro, F.L.A.Machado, F.Pompéia, D.R.Louzada, Patent, WO/2010/094096, February 12, 2010.

[20] W.Andrä, H.Nowak, Magnetism in Medicine: A Handbook, WILEY-VCH Verlag GmbH \& Co. KGaA, Weinheim, 2007, ISBN 978-3-527-40558-9.

[21] J.Clarke, A.I.Braginski, The SQUID Handbook, WILEY-VCH Verlag GmbH, Weinheim, 2006, ISBN 978-3-527-40408-7.

[22] L.T.Bruton, RC-Active Circuits: Theory and Design, Prentice Hall International, London, 1980, ISBN 978-0-137-53467-8. 